\title{
Postprandial glucose, insulin and glucagon-like peptide 1 responses to sucrose ingested with berries in healthy subjects
}

\author{
Riitta Törrönen ${ }^{1 *}$, Essi Sarkkinen ${ }^{2}$, Tarja Niskanen ${ }^{2}$, Niina Tapola ${ }^{2}$, Kyllikki Kilpi ${ }^{3}$ and Leo Niskanen ${ }^{4,5}$ \\ ${ }^{1}$ Department of Clinical Nutrition, Food and Health Research Centre, Institute of Public Health and Clinical Nutrition, \\ University of Eastern Finland, PO Box 1627, FI-70211 Kuopio, Finland \\ ${ }^{2}$ Foodfiles Limited, Kuopio, Finland \\ ${ }^{3}$ Finnsugar Limited, Kantvik, Finland \\ ${ }^{4}$ Department of Internal Medicine, Central Hospital of Central Finland, Jyväskylä, Finland \\ ${ }^{5}$ Institute of Clinical Medicine, University of Eastern Finland, Kuopio, Finland \\ (Submitted 1 December 2010 - Final revision received 6 June 2011 - Accepted 15 July 2011 - First published online 20 September 2011)
}

\section{Abstract}

Berries are often consumed with sucrose. They are also rich sources of polyphenols which may modulate glycaemia after carbohydrate ingestion. The present study investigated the postprandial glucose, insulin and glucagon-like peptide 1 (GLP-1) responses to sucrose ingested with berries, in comparison with a similar sucrose load without berries. A total of twelve healthy subjects were recruited to a randomised, single-blind, placebo-controlled crossover study. They participated in two meal tests on separate days. The berry meal was a purée $(150 \mathrm{~g})$ made of bilberries, blackcurrants, cranberries and strawberries with $35 \mathrm{~g}$ sucrose. The control meal included the same amount of sucrose and available carbohydrates in water. Fingertip capillary and venous blood samples were taken at baseline and at 15, 30, 45, 60, 90 and $120 \mathrm{~min}$ after starting to eat the meal. Glucose, insulin and GLP-1 concentrations were determined from the venous samples, and glucose also from the capillary samples. Compared to the control meal, ingestion of the berry meal resulted in lower capillary and venous plasma glucose and serum insulin concentrations at $15 \mathrm{~min}(P=0 \cdot 021, P<0 \cdot 007$ and $P=0 \cdot 028$, respectively), in higher concentrations at $90 \mathrm{~min}(P=0.028, P=0.021$ and $P=0.042$, respectively), and in a modest effect on the GLP-1 response $(P=0.05)$. It also reduced the maximum increases of capillary and venous glucose and insulin concentrations $(P=0 \cdot 009, P=0 \cdot 011$ and $P=0.005$, respectively), and improved the glycaemic profile $(P<0.001$ and $P=0.003$ for capillary and venous samples, respectively). These results suggest that the glycaemic control after ingestion of sucrose can be improved by simultaneous consumption of berries.

Key words: Berries: Sucrose: Glucose: Insulin

Recent advances in scientific understanding of the multiple health benefits of berry consumption provide strong evidence on berries as excellent food choices in a balanced diet ${ }^{(1)}$. Berries contain essential nutrients such as antioxidant vitamins, minerals and fibre, and are low in energy. They are also rich in bioactive phytochemicals that provide health benefits beyond basic nutrition. Among commonly consumed plant foods, berries are superior sources of numerous polyphenols including anthocyanins and other flavonoids, proanthocyanidins and ellagitannins ${ }^{(2-4)}$.

During industrial or domestic processing, sugar is usually added to mask the acidic taste caused by organic acids and a low content of natural sugars present in berries. Sugar is also needed for preservation of berry products. Although sugar is an integral part of many berry products, it may compromise the beneficial properties of berries, e.g. by inducing postprandial hyperglycaemia and hyperinsulinaemia. Few studies have been dedicated to understand how the addition of sugar to a fruit product affects the glycaemic response.

Dietary polyphenols may influence sucrose metabolism at many levels, as reviewed by Hanhineva $e t a l .{ }^{(5)}$. One of the possible mechanisms is inhibition of digestion and absorption thus modulating postprandial glycaemia. Many polyphenols have inhibited intestinal $\alpha$-glucosidase (maltase/sucrase) activity or glucose transport in vitro, and suppressed the elevation of blood glucose concentration after oral administration of glucose or maltose in animal models ${ }^{(5)}$. Studies involving human subjects have suggested that dietary sources of polyphenols such as apple juice, red wine, decaffeinated coffee and cinnamon may improve short-term glycaemic control ${ }^{(5)}$.

Abbreviations: AUC, area under the curve; CPIR, cephalic phase insulin release; GLP-1, glucagon-like peptide 1.

*Corresponding author: Dr R. Törrönen, fax +358 17 162792, email riitta.torronen@uef.fi 
Current evidence on the effects of berries on postprandial glycaemia is limited. Polyphenol-rich extracts of blueberries, blackcurrants, strawberries and raspberries have inhibited $\alpha$-glucosidase activity in vitro ${ }^{(6,7)}$. In human subjects, cranberry juice sweetened with high-fructose corn syrup induced a different (but not statistically significant) pattern of postprandial glycaemia compared to a similar amount of the sweetener in water ${ }^{(8)}$. Sea buckthorn berries suppressed the peak postprandial insulin concentration and stabilised the glycaemic response after a high-glucose meal ${ }^{(9)}$.

In our previous study ${ }^{(10)}$, berries attenuated the postprandial glucose response to sucrose in healthy subjects. In the present study, we determined the glycaemic profile ${ }^{(11)}$ for sucrose consumed with and without berries. In addition, to obtain more information on the influence of berries on postprandial regulation of glucose homeostasis, insulin and glucagon-like peptide 1 (GLP-1) responses were examined.

\section{Experimental methods}

\section{Subjects}

A total of fifteen volunteers were recruited from the register of Foodfiles Limited in the area of Kuopio, Eastern Finland. At the screening visit, the health status of the subjects was checked by using a structured interview on previous and current diseases, current medication, alcohol and tobacco consumption and the use of food supplements, and by anthropometric measurements and routine blood chemistry (fasting plasma glucose, blood count, serum thyroid-stimulating hormone, creatinine and $\gamma$-glutamyl transferase). Two healthy men and ten women, aged 39-68 years (58 (SD 11) years), with normal weight or slight overweight $(24.3$ (SD $2 \cdot 2) \mathrm{kg} / \mathrm{m}^{2}$, range $20 \cdot 5-27 \cdot 8 \mathrm{~kg} / \mathrm{m}^{2}$ ) and normal fasting plasma glucose $(5 \cdot 4(\mathrm{sD} 0 \cdot 2) \mathrm{mmol} / \mathrm{l}$, range $5 \cdot 0-5 \cdot 8 \mathrm{mmol} / \mathrm{l})$ were eligible for the present study. This study was conducted according to the guidelines laid down in the Declaration of Helsinki and all procedures involving human subjects were approved by the Research Ethics Committee of the Hospital District of Northern Savo (Finland). Written informed consent was obtained from all subjects. The present study has been registered in Current Controlled Clinical Trials, ISRCTN03619857.

\section{Study design}

The randomised, controlled, crossover study was carried out single-blinded for the study nurse and laboratory analyses. Each subject participated in two $2 \mathrm{~h}$ meal tests on separate visits, at least $5 \mathrm{~d}$ apart. The test meals were served in a randomised order in an open-label design. The subjects were instructed to keep their diet, body weight and living habits constant during the study, and to refrain from intensive physical activity on the day before the test and abstain from alcohol for $2 \mathrm{~d}$ before the test. In the evening before the test, they were advised to avoid berries and chocolate, and to consume a meal of choice and repeat that meal before the second test. Smoking was prohibited in the morning before and during the test. Body weight was recorded at both visits.
The tests were carried out between 07.00 and 10.00 hours after a $10-12 \mathrm{~h}$ overnight fast. Baseline blood samples were taken within $20 \mathrm{~min}$ before the test meal and at 15, 30, 45, 60, 90 and $120 \mathrm{~min}$ after starting to eat the meal for measurements of plasma glucose, serum insulin and plasma GLP-1 concentrations. The subjects were advised to consume the test meals within $15 \mathrm{~min}$.

\section{Blood samples and analyses}

Venous blood samples were collected through an indwelling cannula inserted into a forearm vein. The samples for plasma glucose were collected in citrate-fluoride tubes and for plasma GLP-1 in EDTA tubes containing aprotinin (Becton-Dickinson, Franklin Lakes, NJ, USA). The samples were frozen immediately after separation of plasma or serum, and the glucose and insulin samples were stored at $-20^{\circ} \mathrm{C}$ and GLP-1 samples at $-70^{\circ} \mathrm{C}$ until analysis. Glucose concentrations were analysed with a hexokinase method (Advia ${ }^{\circledR}$ Chemistry System; Siemens Medical Solutions Diagnostics, Tarrytown, NY, USA), insulin with an immunoluminometric assay (Immulite ${ }^{\circledR} 2500$ Immunoassay System; Siemens Healthcare Diagnostics, Deerfield, IL, USA), and GLP-1 with a competitive enzyme immunoassay specific for the active forms GLP-1 7-36 amide and 7-37 (Millipore Corporation, Billerica, MA, USA). In addition, plasma glucose concentration was also determined from a fingertip capillary blood drop taken just before the venous samples by using a HemoCue Glucose $201^{+}$analyzer (HemoCue AB, Angelholm, Sweden) calibrated to plasma-equivalent glucose concentrations.

\section{Test meals}

The study included a berry meal and a control meal without berries. The berry meal included a mixed berry purée (150 g) with $35 \mathrm{~g}$ added sucrose and $120 \mathrm{ml}$ of tap water. The mixed berry purée consisted of blackcurrants (Ribes nigrum), bilberries (wild European blueberries, Vaccinium myrtillus), cranberries (Vaccinium oxycoccos) and strawberries (Fragaria $\times$ ananassa), $37.5 \mathrm{~g}$ of each berry. The natural sugar composition of the purée was $2.9 \%$ glucose $(4.4 \mathrm{~g} /$ portion) and $3.1 \%$ fructose ( $4.7 \mathrm{~g} /$ portion), as analysed by HPLC. The portion of the berry purée was thawed and $35 \mathrm{~g}$ of sucrose was added just before the test meal was served. The control meal included $250 \mathrm{ml}$ of tap water with $35 \mathrm{~g}$ sucrose, $4.4 \mathrm{~g}$ glucose and $4.7 \mathrm{~g}$ fructose to achieve the similar profile and amounts of available carbohydrates as provided by the berry meal. The nutrient composition of the test meals is given in Table 1.

\section{Calculations and statistical analyses}

The data were analysed by using the SPSS 17.0 for Windows statistical program (SPSS, Inc., Chicago, IL, USA), and the results are expressed as means and standard deviations with $P$ values less than 0.05 considered to be statistically significant. Normal distribution of the variables was checked with the Shapiro-Wilk test before further analyses. The statistical 
Table 1. Nutrient composition of the test meals

\begin{tabular}{lcc}
\hline & Berry meal & Control meal \\
\hline Sucrose $(g)^{\star}$ & 35 & 35 \\
Glucose $(g) \dagger$ & 4.4 & 4.4 \\
Fructose $(g) \dagger$ & 4.7 & 4.7 \\
Total fibre $(g) \ddagger$ & 5.4 & 0 \\
Insoluble fibre $(g) \ddagger$ & 3.7 & 0 \\
Fat $(g) \ddagger$ & 0.7 & 0 \\
Protein $(g) \ddagger$ & 0.9 & 0 \\
\hline
\end{tabular}

* Added to both meals.

† Analysed from the berry purée and added to the control meal.

$\ddagger$ Data from the Finnish food composition database (Fineli ${ }^{\circledR}$, Release 11, National Institute for Health and Welfare; www.fineli.fi).

significance of the overall difference in normally distributed variables between the meals was assessed with a general linear model for repeated measures followed by pairedsamples $t$ tests with Bonferroni correction to analyse the differences between the test meals at different time points. The maximum increases from the baseline concentrations were calculated by subtracting the fasting value from the highest value, and the differences between the test meals were analysed by paired-samples $t$ tests. The $0-120 \mathrm{~min}$ incremental area under the curve (AUC) were calculated for each subject and meal by using Canvas 8.0.2 (Deneba Software, Miami, FL, USA), ignoring the area below the baseline ( $0 \mathrm{~min}$ ) concentration. The Canvas program was also used for calculation of glycaemic profiles for each subject and test meal according to Rosén et al. ${ }^{(11)}$, by dividing the time ( $\mathrm{min}$ ) during which the plasma glucose was above the fasting concentration with the incremental peak value $(\mathrm{mmol} / \mathrm{l})$. The statistical significance of the differences in normally and non-normally distributed AUC and glycaemic profiles was assessed with paired-sample $t$ test or Wilcoxon test, respectively.

\section{Results}

There were no significant changes in body weight during the study. The mean body weight was 66.6 (SD 10.5) kg at the first visit and 66.9 (SD 10.8) $\mathrm{kg}$ at the second visit. The fasting capillary and venous plasma glucose, serum insulin and plasma GLP-1 concentrations did not differ between the test meal occasions. The berry meal was ingested in 8.25 (SD 1.85) min and the control meal in 8.83 (SD 1.10) min. The ingestion order of the meals had no effect on the results.

\section{Glucose}

The ingestion of the berry meal resulted in significantly lower capillary $(P=0.021)$ and venous $(P<0.007)$ plasma glucose responses in the early postprandial phase at $15 \mathrm{~min}$ time point than the control meal and a nearly significantly lower capillary plasma glucose response at $30 \mathrm{~min} \quad(P=0.084$; Fig. 1). Furthermore, the capillary $(P=0.028)$ and venous $(P=0.021)$ glucose responses at $90 \mathrm{~min}$ after starting the berry meal were significantly higher than after the control meal. The maximum increases in capillary and venous glucose concentrations were almost $30 \%$ lower after ingestion of the berry meal compared to the control meal (Table 2).

After the berry meal, the mean AUC (0-120 min) of plasma glucose responses were smaller than the AUC after the control meal, but the differences did not reach statistical significance (Table 2). However, the differences in the glycaemic profiles were statistically significant $(P<0.001$ for capillary plasma glucose, $P=0.003$ for venous plasma glucose). The values for glycaemic profiles were doubled after the berry meal compared to the control meal (Table 2).

\section{Insulin}

Compared to the control meal, the berry meal resulted in a significantly lower serum insulin concentration at $15 \mathrm{~min}$ $(P=0 \cdot 028)$ and significantly higher concentrations at 90 and $120 \mathrm{~min}$ ( $P=0.042$ for both; Fig. 2). The maximum increase from the baseline was approximately $20 \%$ lower after ingestion of the berry meal compared to the control meal (Table 2). No difference was observed in the AUC.
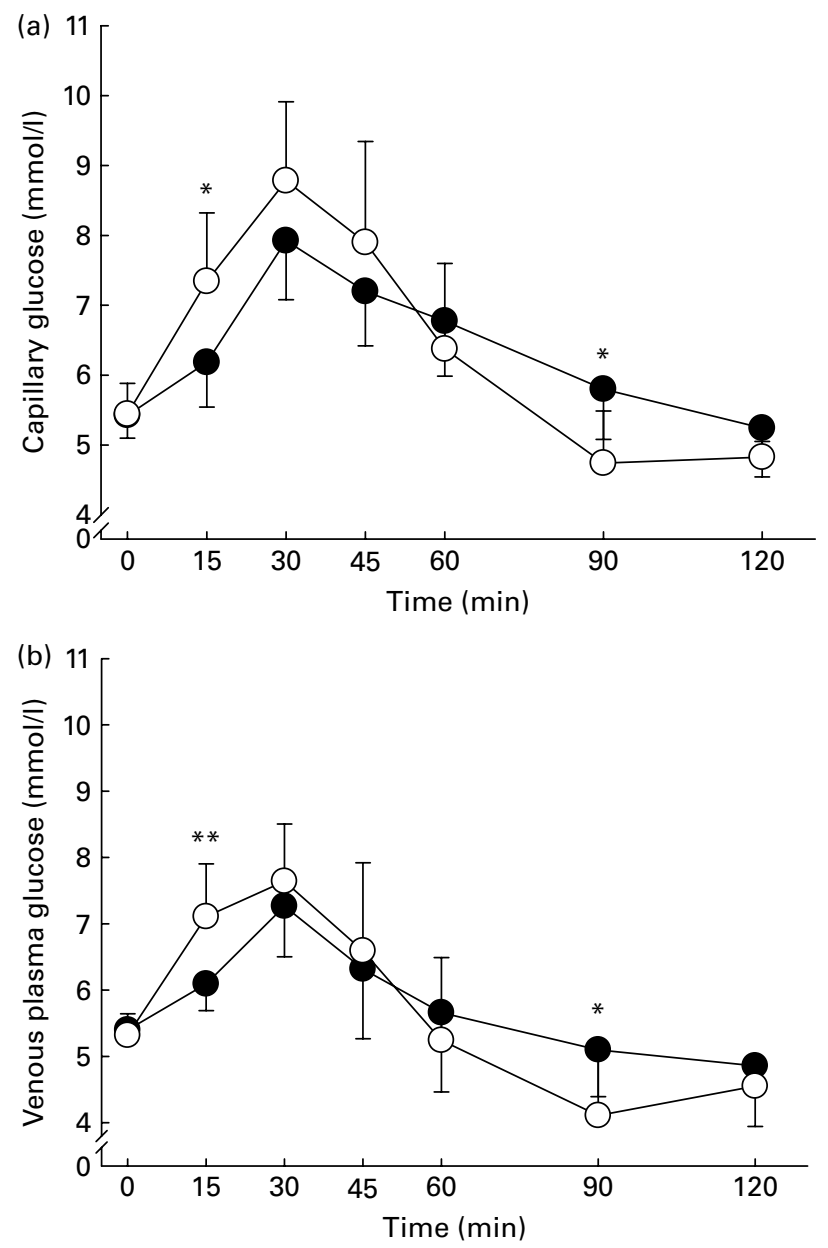

Fig. 1. (a) Capillary and (b) venous plasma glucose concentrations after ingestion of the berry meal $(\bullet)$ and the control meal $(O)$. Values are means with their standard deviations represented by vertical bars ( $n$ 12). Overall mean values were significantly different between the meals (a) $P=0.001$ and (b) $P=0.002$ (general linear model for repeated measures). Mean values were significantly different between meals: ${ }^{\star} P<0.05$, ${ }^{\star \star} P<0.01$ (pairedsamples $t$ test with Bonferroni correction). 
Table 2. Maximum increases from the baseline and area under the curve (AUC) for glucose, insulin and glucagon-like peptide 1 (GLP-1) responses and glycaemic profiles after the test meals (Mean values and standard deviations)

\begin{tabular}{|c|c|c|c|c|c|}
\hline & \multicolumn{2}{|c|}{ Berry meal } & \multicolumn{2}{|c|}{ Control meal } & \multirow[b]{2}{*}{$P^{*}$} \\
\hline & Mean & SD & Mean & SD & \\
\hline \multicolumn{6}{|l|}{ Maximum increase } \\
\hline Capillary glucose (mmol/l) & $2 \cdot 5$ & 0.7 & 3.5 & $1 \cdot 0$ & 0.009 \\
\hline Venous glucose $(\mathrm{mmol} / \mathrm{l})$ & 1.9 & 0.9 & $2 \cdot 7$ & 0.9 & 0.011 \\
\hline Insulin (pmol/l) & 115 & 49 & 145 & 46 & 0.005 \\
\hline GLP-1 (pmol//) & 8.7 & $10 \cdot 2$ & $6 \cdot 0$ & 5.9 & NS \\
\hline \multicolumn{6}{|l|}{ AUC $0-120 \mathrm{~min}$} \\
\hline Capillary glucose $(\min \times \mathrm{mmol} / \mathrm{l})$ & 121 & 36 & 137 & 53 & NS \\
\hline Venous glucose $(\mathrm{min} \times \mathrm{mmol} / \mathrm{l})$ & 72 & 50 & 91 & 56 & NSt \\
\hline Insulin $(\min \times \mathrm{pmol} / \mathrm{l})$ & 7152 & 2208 & 7236 & 1938 & NS \\
\hline GLP-1 $(\min \times \mathrm{pmol} / \mathrm{l})$ & 312 & 345 & 216 & 289 & NS \\
\hline \multicolumn{6}{|l|}{ Glycaemic profile } \\
\hline Capillary glucose $(\mathrm{min} /(\mathrm{mmol} / \mathrm{l}))$ & $46 \cdot 7$ & 24.8 & 22.6 & 8.5 & $<0.001$ \\
\hline Venous glucose $(\mathrm{min} /(\mathrm{mmol} / \mathrm{l}))$ & 49.9 & 21.1 & 24.7 & $9 \cdot 3$ & 0.003 \\
\hline
\end{tabular}

\section{Glucagon-like peptide 1}

The overall difference in GLP-1 concentrations between the meals was nearly statistically significant $(P=0.05)$, and the concentrations tended to be higher during $1 \mathrm{~h}$ after the berry meal than after the control meal (Fig. 3). Also, the maximum increase from the baseline and the mean AUC of GLP-1 response tended to be approximately $45 \%$ higher after the berry meal, but these differences were not statistically significant (Table 2).

\section{Discussion}

The present study confirms the findings of our previous study ${ }^{(10)}$ showing beneficial effects of berries on postprandial glycaemia induced by sucrose. The present and the previous studies found a significantly lower glucose response at $15 \mathrm{~min}$ after starting the berry meal and significantly higher response at $90 \mathrm{~min}$ compared to the control meal. In the previous study, there was also a significant difference in the capillary plasma glucose concentration $(1.2 \mathrm{mmol} / \mathrm{l})$ at $30 \mathrm{~min}^{(10)}$. In the present study, the capillary plasma glucose concentration $30 \mathrm{~min}$ after the berry meal was $0.9 \mathrm{mmol} / \mathrm{l}$ lower than after the control meal. However, this difference was not statistically significant.

In the present study, we determined plasma glucose responses from both capillary and venous samples. The venous plasma glucose concentrations, maximum increases from the baseline and AUC were lower than those measured from the capillary samples, as reported earlier for other carbohydrate-rich foods ${ }^{(12,13)}$. According to Brouns et al. ${ }^{(12)}$, the sensitivity of glucose measurement is greater using capillary blood sampling. In the present study, however, the differences in the glucose responses between the test meals were detectable also from venous blood samples.

There was a clear difference in the shape of the glucose curve, with an attenuated elevation in the early postprandial phase and a less rapid fall below the fasting level after the berry meal. As a consequence of the less rapid fall, there was no statistically significant difference in the $0-120 \mathrm{~min}$ AUC between the test meals, as was also the case in our previous study ${ }^{(10)}$. In order to illustrate the course of the postprandial glycaemia, Rosén et $a l .{ }^{(11)}$ introduced a new quantitative parameter, the glycaemic profile. It is defined as the duration of the incremental postprandial blood glucose response divided by the blood glucose incremental peak. A high value of glycaemic profile indicates facilitated postprandial glycaemic regulation, with a lower glucose peak and a less pronounced hypoglycaemia. We calculated the glycaemic profile for our test meals and observed significantly higher values for the berry meal (measured either from capillary or venous samples). Thus, ingestion of berries

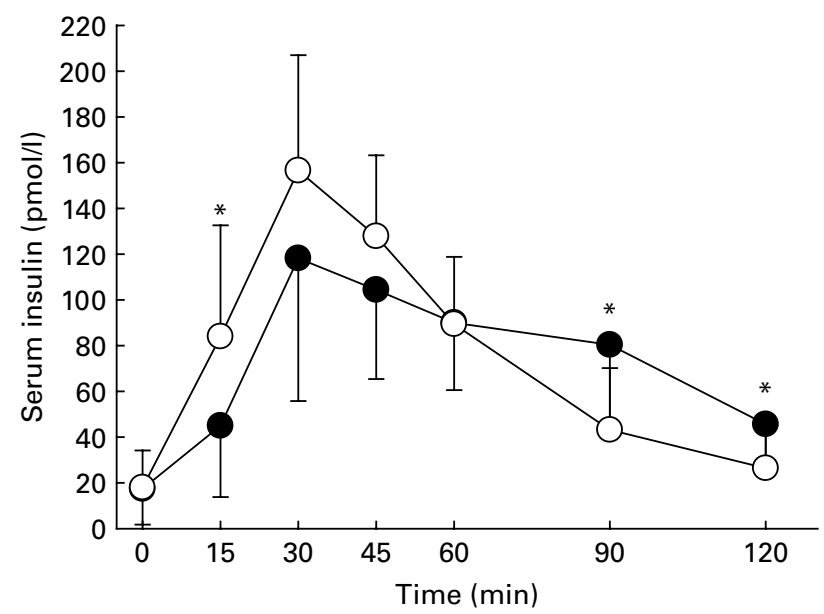

Fig. 2. Serum insulin concentrations after ingestion of the berry meal (•) and the control meal $(\bigcirc)$. Values are means with their standard deviations represented by vertical bars $(n 12)$. Overall mean values were significantly different between the meals $(P=0.001$, general linear model for repeated measures). *Mean values were significantly different between meals $(P<0.05$, paired-samples $t$ test with Bonferroni correction). 


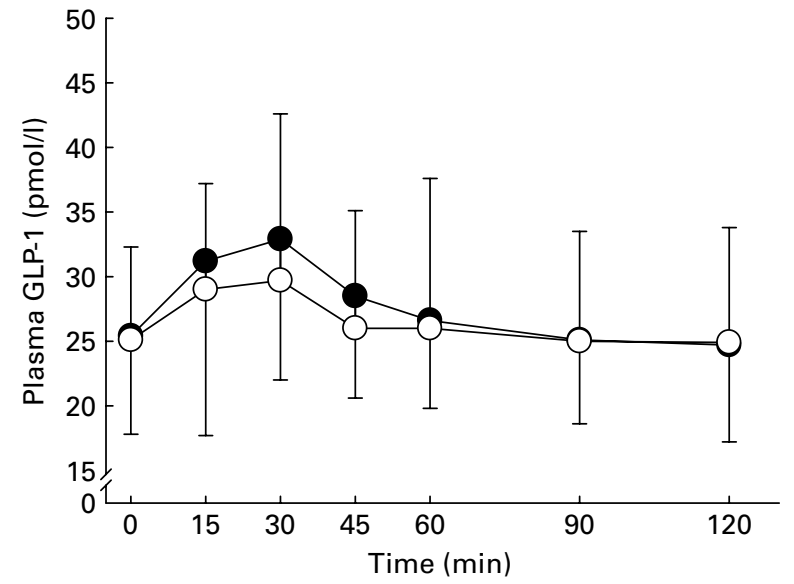

Fig. 3. Plasma glucagon-like peptide $1(\mathrm{GLP}-1)$ concentrations after ingestion of the berry meal $(\bullet)$ and the control meal $(\bigcirc)$. Values are means with their standard deviations represented by vertical bars $(n 12)$. Overall mean values were significantly different between the meals $(P=0.05$, general linear model for repeated measures).

with sucrose clearly improved the postprandial glycaemic regulation compared to sucrose alone.

Meals high in available carbohydrates such as sucrose induce early postprandial hyperglycaemia, which stimulates insulin secretion resulting in hyperinsulinaemia. Repeated postprandial hyperglycaemia and hyperinsulinaemia may cause insulin resistance, $\beta$-cell dysfunction and inflammation $^{(14,15)}$. An exaggerated postprandial insulin response may cause transient late postprandial hypoglycaemia, activating the glucocorticoid axis and eliciting secretion of counter-regulatory stress hormones ${ }^{(14)}$. In the present study, the serum insulin concentrations paralleled plasma glucose concentrations. The berry meal induced a delayed and attenuated glucose response which stimulated a lower and prolonged insulin response compared to the control meal, and no signs of exaggerated insulin response were seen. Thus, consumption of berries with sucrose resulted in a more beneficial insulin response than did sucrose alone.

The berry meal tended to enhance postprandial GLP-1 concentrations slightly more than the control meal did, with the overall difference close to statistical significance. Due to the large interindividual variations typical for postprandial GLP-1 responses, the differences between the meals at any of the time points were not statistically significant. Therefore, the evidence for the effect of the berry meal is weaker for GLP-1 than for glucose and insulin responses.

GLP-1 is an incretin hormone secreted from the L-cells in the distal small intestine and colon in response to food ingestion $^{(16)}$. It is rapidly released after meals, and responds to the presence of carbohydrates or other macronutrients in the intestinal lumen ${ }^{(17)}$. Its main action is to stimulate glucose-dependent insulin secretion when glucose is absorbed through the gastrointestinal tract and the blood glucose concentration is above the normal physiological range ${ }^{(16,18)}$. When the glucose concentration has returned to the basal level, GLP-1 no longer stimulates insulin secretion and thus does not induce hypoglycaemia. GLP-1 also regulates a number of other processes that reduce fluctuations in blood glucose concentrations, such as glucagon secretion, gastric emptying, appetite and food intake. Therefore, it has an important role in the maintenance of postprandial glucose homeostasis.

In the present study, the increase in GLP-1 concentrations after the berry meal did not stimulate peak postprandial insulin release. The insulin-stimulating effect of GLP-1 depends on the amount of glucose ingested, being small with $25 \mathrm{~g}$ and very large with $100 \mathrm{~g}$ of glucose ${ }^{(16)}$. The berry meal contained $17 \cdot 5 \mathrm{~g}$ glucose from sucrose and $4.4 \mathrm{~g}$ natural glucose from the berries. The glucose challenge provided by the berry meal was probably too small to evoke a GLP-1-mediated peak increase in insulin release. However, it may have contributed to the more prolonged insulin release and thus promoted smoother handling of the glucose load.

Our results are in agreement with previous studies which investigated the effects of polyphenol-rich apple juice ${ }^{(19)}$, coffee $^{(20)}$ or cinnamon ${ }^{(21)}$ on GLP-1 response. The postprandial GLP-1 concentrations were elevated when glucose was consumed in apple juice or decaffeinated coffee or rice pudding consumed with cinnamon, indicating that polyphenols may contribute to enhanced GLP-1 secretion.

Based on our previous data, we have estimated that the berry meal provided nearly $800 \mathrm{mg}$ polyphenols, including anthocyanins, flavonols, phenolic acids, proanthocyanidins and ellagitannins ${ }^{(10)}$. As reviewed by Hanhineva et al. ${ }^{(5)}$, several in vitro studies have shown that these polyphenols inhibit $\alpha$-glucosidase activity and intestinal uptake of glucose mediated by active Na-dependent transport via Na glucose cotransporter 1 and facilitated Na-independent transport via GLUT2. In addition, polyphenol-rich extracts of blackcurrants, blueberries and strawberries, the constituents of our berry meal, have been shown to inhibit $\alpha$-glucosidase activity in vitro ${ }^{(6,7)}$. Therefore, reduced digestion of sucrose by inhibition of $\alpha$-glucosidase and/or slower absorption of the liberated glucose due to polyphenols are the potential mechanisms underlying the delayed and suppressed postprandial glucose and insulin responses observed after consumption of the berry meal. These mechanisms could also explain the slightly augmented GLP-1 response. Impaired digestion and/or absorption result in a higher level of sugars in the intestinal lumen and consequent stimulation of GLP-1 release. Interestingly, reduced glucose and insulin responses as well as augmented GLP-1 responses have been reported when sucrose was ingested with a known $\alpha$-glucosidase inhibitor, acarbose ${ }^{(22,23)}$.

Besides polyphenols, also dietary fibre may potentially have affected the postprandial responses to the berry meal. Postprandial glucose and insulin responses are influenced by fibre-rich meals, depending on the source and dose of soluble and total fibre ${ }^{(24)}$. Based on the Finnish food composition database (Fineli ${ }^{\circledR}$, Release 11, National Institute for Health and Welfare; www.fineli.fi), we have calculated that the berry meal provided $5.4 \mathrm{~g}$ of total fibre and no more than $1.7 \mathrm{~g}$ of soluble fibre (Table 1 ). We propose that it is unlikely that this relatively small amount of fibre could solely explain the changes observed in the glucose and insulin responses. The effect of sea buckthorn berries on postprandial insulin 
response to a high-glucose meal was not associated with the insoluble fibre fraction ${ }^{(9)}$. Dietary fibre may also modulate postprandial GLP-1 response. Elevated, blunted and unaffected responses have been reported, possibly related to the type and amount of fibre ${ }^{(17)}$. Due to the limited evidence currently available, it is difficult to draw any conclusions on the potential contribution of the fibre content of the berry meal to the GLP-1 response.

The third aspect to be considered is the semi-solid consistency of the berry meal which may have affected the glycaemic response by delaying gastric emptying compared to the liquid control meal. For our test meals, the gastric emptying rate could be influenced by the volume, energy content and presence of soluble fibre ${ }^{(25)}$. The volume of the meals and the content of available carbohydrates were adjusted to be similar. The amounts of fat and protein were very small $(\leq 1 \mathrm{~g})$ in the berry meal, having a negligible contribution to the energy content and gastric emptying. Due to the soluble fibre, the viscosity is expected to be higher in the berry meal than in the control meal. However, as discussed above, the amount of viscosity-producing soluble fibre was relatively small. The role of polyphenols in gastric emptying is obscure. The results on the effect of polyphenol-rich cinnamon on gastric emptying rate are inconsistent ${ }^{(21,26)}$. We suggest that the consistency may be one of the favourable properties of the berry puree contributing to the improved postprandial glycaemia, but not likely to fully explain the beneficial responses. The differences in the glucose and insulin responses cannot be explained by the time taken by the ingestion of the meals, since the average time needed for ingestion of the berry meal was slightly shorter than for the control meal.

Since we found statistically significant differences in glucose and insulin responses as early as $15 \mathrm{~min}$ after onset of the meals, it is possible that neurally mediated preabsorptive mechanisms were involved. Oral sensory stimulation may elicit cephalic phase insulin release (CPIR) before glucose absorption $^{(27,28)}$. In response to masticating and tasting food, the pancreas rapidly begins to secrete insulin. This initial pulse of insulin secretion is followed by much larger, postabsorptive sustained insulin secretion measured herein. The CPIR thus anticipates and mimics, at an attenuated level, the postabsorptive insulin response to changes in blood glucose concentration. Despite the magnitude of the CPIR being low, just detectable, it plays a significant physiological role preparing the body for the more efficient handling of the nutrients ${ }^{(29)}$. CPIR is mainly mediated by vagal efferent activation, and it typically peaks at $4 \mathrm{~min}$ after oral sensory stimulation and returns to baseline by $10 \mathrm{~min}$ poststimulation $^{(27,28)}$. Our test meals with clearly different taste and texture properties presumably elicited different sensory stimulation in the oral cavity. Therefore, also differences in CPIR may be expected, and the lowered postprandial glucose and insulin responses after the berry meal may be partly explained by more efficient CPIR resulting in more efficient postload glucose handling. Since our study was not designed to detect the very early responses, it is not possible to draw any definite conclusions on the contribution of CPIR to our results. In future studies, the CPIR responses in connection with responses to carbohydrates with berries should be determined.

In conclusion, the results of this study show that berries consumed with sucrose improve the postprandial glycaemic control compared to sucrose alone. The purée made of commonly consumed berries had a beneficial impact on glucose, insulin and GLP-1 responses after sucrose ingestion. Glycaemic profile, the new parameter introduced by Rosén et al. ${ }^{(11)}$, was found as a valuable tool for quantification of the beneficial changes in the course of the glucose response. These results are consistent with reduced digestion of sucrose and/or slowed absorption of glucose caused by berry polyphenols. Our findings have practical implications, since berries are often served with sucrose. They encourage further studies to determine the effects of berries with different polyphenol composition on postprandial glycaemic control.

\section{Acknowledgements}

The study was funded by Finnsugar Limited. The authors declare no conflicts of interest. E. S., N. T. and K. K. contributed to the conception and design of the study. T. N. and N. T. carried out the study in practice, including data management and statistics. T. N., E. S. and R. T. wrote the first draft of the manuscript and N. T., L. N. and K. K. critically revised the manuscript.

\section{References}

1. Seeram NP (2010) Recent trends and advances in berry health benefits research. J Agric Food Chem 58, 3869-3870.

2. Koponen JM, Happonen AM, Mattila PH, et al. (2007) Contents of anthocyanins and ellagitannins in selected foods consumed in Finland. I Agric Food Chem 55 , 1612-1619.

3. Ovaskainen ML, Törrönen R, Koponen JM, et al. (2008) Dietary intake and major food sources of polyphenols in Finnish adults. J Nutr 138, 562-566.

4. Hellström JK, Törrönen AR \& Mattila PH (2009) Proanthocyanidins in common food products of plant origin. J Agric Food Chem 57, 7899-7906.

5. Hanhineva K, Törrönen R, Bondia-Pons I, et al. (2010) Impact of dietary polyphenols on carbohydrate metabolism. Int J Mol Sci 11, 1365-1402.

6. McDougall GJ, Shpiro F, Dobson P, et al. (2005) Different polyphenolic components of soft fruits inhibit $\alpha$-amylase and $\alpha$-glucosidase. J Agric Food Chem 53, 2760-2766.

7. da Silva Pinto M, Kwon YI, Apostolidis E, et al. (2008) Functionality of bioactive compounds in Brazilian strawberry (Fragaria $\times$ ananassa Duch.) cultivars: evaluation of hyperglycemia and hypertension potential using in vitro models. J Agric Food Chem 56, 4386-4392.

8. Wilson T, Singh AP, Vorsa N, et al. (2008) Human glycemic response and phenolic content of unsweetened cranberry juice. J Med Food 11, 46-54.

9. Lehtonen H-M, Järvinen R, Linderborg K, et al. (2010) Postprandial hyperglycemia and insulin response are affected by sea buckthorn (Hippophaë rhamnoides ssp. turkestanica) berry and its ethanol-soluble metabolites. Eur J Clin Nutr 64 , 1465-1471. 
10. Törrönen R, Sarkkinen E, Tapola N, et al. (2010) Berries modify the postprandial plasma glucose response to sucrose in healthy subjects. BrJ Nutr 103, 1094-1097.

11. Rosén LAH, Blanco Silva LO, Andersson UK, et al. (2009) Endosperm and whole grain rye breads are characterized by low post-prandial insulin response and a beneficial blood glucose profile. Nutr J 8, 42 .

12. Brouns F, Bjorck I, Frayn KN, et al. (2005) Glycaemic index methodology. Nutr Res Rev 18, 145-171.

13. Hätönen KA, Similä ME, Virtamo JR, et al. (2006) Methodologic considerations in the measurement of glycemic index: glycemic response to rye bread, oatmeal porridge, and mashed potato. Am J Clin Nutr 84, 1055-1061.

14. Ludwig DS (2002) The glycemic index. Physiological mechanisms relating to obesity, diabetes, and cardiovascular disease. JAMA 287, 2414-2423.

15. Dandona P, Aljada A, Chaudhuri A, et al. (2005) Metabolic syndrome. A comprehensive perspective based on interactions between obesity, diabetes, and inflammation. Circulation 111, 1448-1454.

16. Holst JJ (2007) The physiology of glucagon-like peptide 1. Physiol Rev 87, 1409-1439.

17. Karhunen LJ, Juvonen KR, Huotari A, et al. (2008) Effect of protein, fat, carbohydrate and fibre on gastrointestinal peptide release in humans. Regul Pept 149, 70-78.

18. D'Alessio DA \& Vahl TP (2004) Glucagon-like peptide 1: evolution of an incretin into a treatment for diabetes. $\mathrm{Am}$ J Physiol Endocrinol Metab 286, E882-E890.

19. Johnston KL, Clifford MN \& Morgan LM (2002) Possible role for apple juice phenolic compounds in the acute modification of glucose tolerance and gastrointestinal hormone secretion in humans. J Sci Food Agric 82, 1800-1805.

20. Johnston KL, Clifford MN \& Morgan LM (2003) Coffee acutely modifies gastrointestinal hormone secretion and glucose tolerance in humans: glycemic effects of chlorogenic acid and caffeine. Am J Clin Nutr 78, 728-733.

21. Hlebowicz J, Hlebowicz A, Lindstedt S, et al. (2009) Effects of 1 and $3 \mathrm{~g}$ cinnamon on gastric emptying, satiety, and postprandial blood glucose, insulin, glucose-dependent insulinotropic polypeptide, glucagon-like peptide 1, and ghrelin concentrations in healthy subjects. Am J Clin Nutr 89, 815-821.

22. Ranganath L, Norris F, Morgan L, et al. (1998) Delayed gastric emptying occurs following acarbose administration and is a further mechanism for its anti-hyperglycaemic effect. Diabet Med 15, 120-124.

23. Seifarth C, Bergmann J, Holst JJ, et al. (1998) Prolonged and enhanced secretion of glucagon-like peptide 1 (7-36 amide) after oral sucrose due to alpha-glucosidase inhibition (acarbose) in type 2 diabetic patients. Diabet Med 15, 485-491.

24. Ulmius M, Johansson A \& Önning G (2009) The influence of dietary fibre source and gender on the postprandial glucose and lipid response in healthy subjects. Eur $J$ Nutr $\mathbf{4 8}$, 395-402

25. Moukarzel AA \& Sabri MT (1996) Gastric physiology and function: effects of fruit juices. J Am Coll Nutr 15, Suppl. 5, 18S-25S.

26. Hlebowicz J, Darwiche G, Björgell O, et al. (2007) Effect of cinnamon on postprandial blood glucose, gastric emptying, and satiety in healthy subjects. Am J Clin Nutr 85, 1552-1556.

27. Teff K (2000) Nutritional implications of cephalic phase responses. Appetite 34, 206-213.

28. Teff KL (2011) How neural mediation of anticipatory and compensatory insulin release helps us tolerate food. Physiol Behav 103, 44-50.

29. Ahrén B \& Holst JJ (2001) The cephalic insulin response to meal ingestion in humans is dependent on both cholinergic and noncholinergic mechanisms and is important for postprandial glycemia. Diabetes 50, 1030-1038. 